Vol. 39(2), pp. 92-103, Dec. 2020

ISSN 1821-536X (print)

ISSN 2619-8789 (electronic)
Tanzania Journal of Engineering and Technology

Copyright () 2020 College of Engineering and

Technology, University of Dar es Salaam

Full Length Research Paper

\title{
Possibilities and Challenges of Radio Frequency Energy Harvesting in Dar es Salaam, Tanzania
}

\author{
*Fredrick Isingo, Prosper Mafole, Abdi Abdalla \\ Department of Electronics \& Telecommunications Engineering, University of Dar es \\ Salaam, Tanzania \\ *Corresponding author: fmisingo@gmail.com
}

\begin{abstract}
This paper presents investigation on the possibilities and challenges of harvesting ambient Radio Frequency Energy (RFE) at Dar es Salaam region in Tanzania. The Radio Frequency $(R F)$ signals were measured using a Rohde and Schwarz FSC 3 spectrum analyzer observing available frequencies with their respective power. Among several RF signals received, the most powerful signals observed were; $800 \mathrm{MHz}, 950 \mathrm{MHz}, 2100 \mathrm{MHz}$ and $2400 \mathrm{MHz}$, having average signal strengths of about $-30.29 \mathrm{dBm},-35.94 \mathrm{dBm},-42.90 \mathrm{dBm}$ and $-30.42 \mathrm{dBm}$ respectively. The power possessed within these frequencies were suitable to be harvested due to their signal strengths, an overall power average of $-34.89 \mathrm{dBm}$ was obtained and a multi narrowband harvester was designed and simulated using real-time values on Keysight's Advanced Design System (ADS) 2019. The simulation results confirm a promising possibility of harvesting RF energy to power ultra-low-power devices in the Internet of Things (IoT) and beyond.
\end{abstract}

Key words: DC-DC Boost, Energy Harvesting, Impedance matching, RF-DC conversion Schottky Diode, Super Capacitor array, Voltage multipliers.

\section{INTRODUCTION}

The gradual demands in ultra-low-power devices are of great interest nowadays, from the inception of the Internet of Things (IoT) (Jose et al., 2015; Ejaz et al., 2017), devices have become smarter, smaller and more energy-efficient (Cansiz et al., 2019). Radio Frequency (RF) energy harvesting, being a major source for providing reliable and sustainable energy is expected to keep Ultra-Low Powered (ULP) devices powered throughout its lifetime. To achieve such promising longevity, there is a need to investigate the possibilities of harvesting the available frequencies so as to enable proper functionality of battery-less systems with zero maintenance (Saini and Baghini, 2019). The RF spectral power density obtained from the spectrum analyser were analysed and simulated in real-time to demonstrate a predictive output based on the feasibility study.

RF energy harvesting sometimes termed as wireless power transfer was initially proposed by Nikola Tesla in the early $20^{\text {th }}$ Century, as one of the most reliable and maintenance-free system (Marincic, 1982; Valenta and Durgin, 2014). The basic structure of any RF energy harvester is comprised of a receiving antenna that accepts a specific frequency band and tunnels its energy to rectifiers that convert 
the signal to DC. More energy can be converted to achieve more output voltages and currents through using extremely sensitive rectifiers as described by Shariati et al. (2015). The energy harvested is thereafter stored in either a battery or supercapacitor awaiting to be used (Olgun et al., 2010).

This work, proposes a new multi narrowband one to one architecture on impedance matching, as well as a newly designed Nano dc to dc boost converter using MAX 17220, which improves output voltage and current. In addition, this work also provides a summary on the $\mathrm{RF}$ intensities available in Dar es Salaam region. The RF analysis and experiments were carried out at the College of Information and Computer Technology (CoICT) campus, University of Dar es Salaam, while data was collected at random places in Dar es Salaam region in Tanzania.

\section{METHODS AND MATERIALS}

\section{Description of Study Area}

Dar es Salaam region was chosen as a study case area for investigative purpose of RF energy harvesting as described in Figure 1. RF data was collected from different parts of the City using a synchronized spectrum analyser and GPS, the later was analysed with Rohde \& Schwatz (R\&S) Instrument view and Quantum Geographic Information System (QGIS) to provide an intuitive RF coverage intensity map.

\section{Tools and Methods}

The RF survey was conducted using the Rhode \& Schwarz FSC 3 Spectrum analyser, Garmin GPS 18x for RF location tags, as in Figure 2. The block diagram describes the equipment layout and connections used for data logging, synchronization and graphical output generation.

The RF density data was collected at random locations within the University's premises; sample data attained are graphically presented in Figure 3 and Figure 4. The setup used for sample RF data collection was as shown in Figure 2. Through keeping the same setup on different locations within Dar es Salaam, a heatmap output expressing location-based radiation intensities will be shown as an output. The average sample data obtained from the RF survey at CoICT campus of the University of Dar es Salaam are expressed on Table 1. The results demonstrate a positive possibility on harvesting the received RF signals at such power levels (Khemar et al., 2018).

The RF to location data log time was set to a 60 second interval in a vehicle collecting RF data around the city. More than 180,000 points were collected across the city and was used for simulation purpose. Table 2 describes the location with its RF signal power in $\mathrm{dBm}$. 


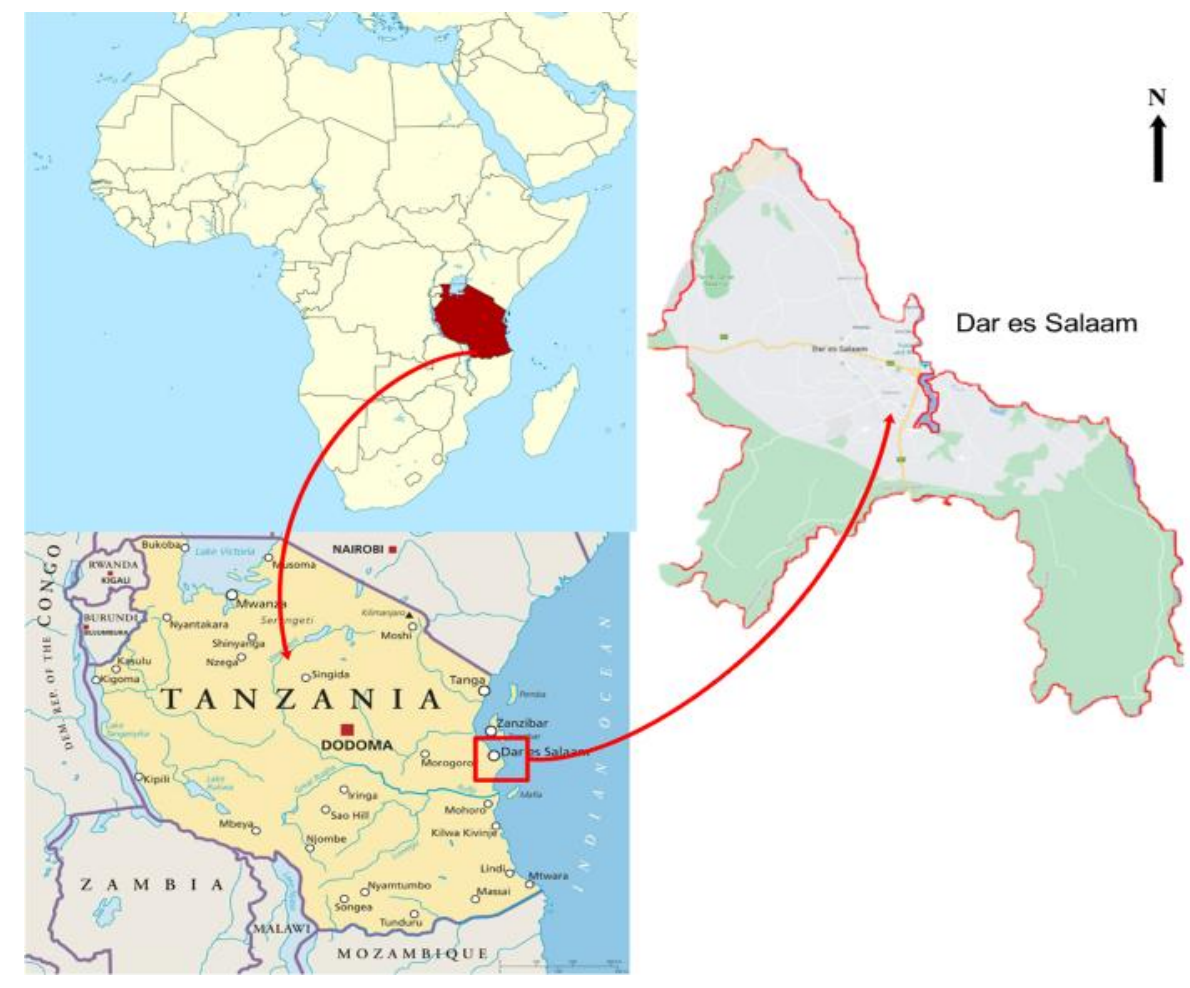

Figure 1: Dar es Salaam Region (Source: QGIS Development Team, 2020)

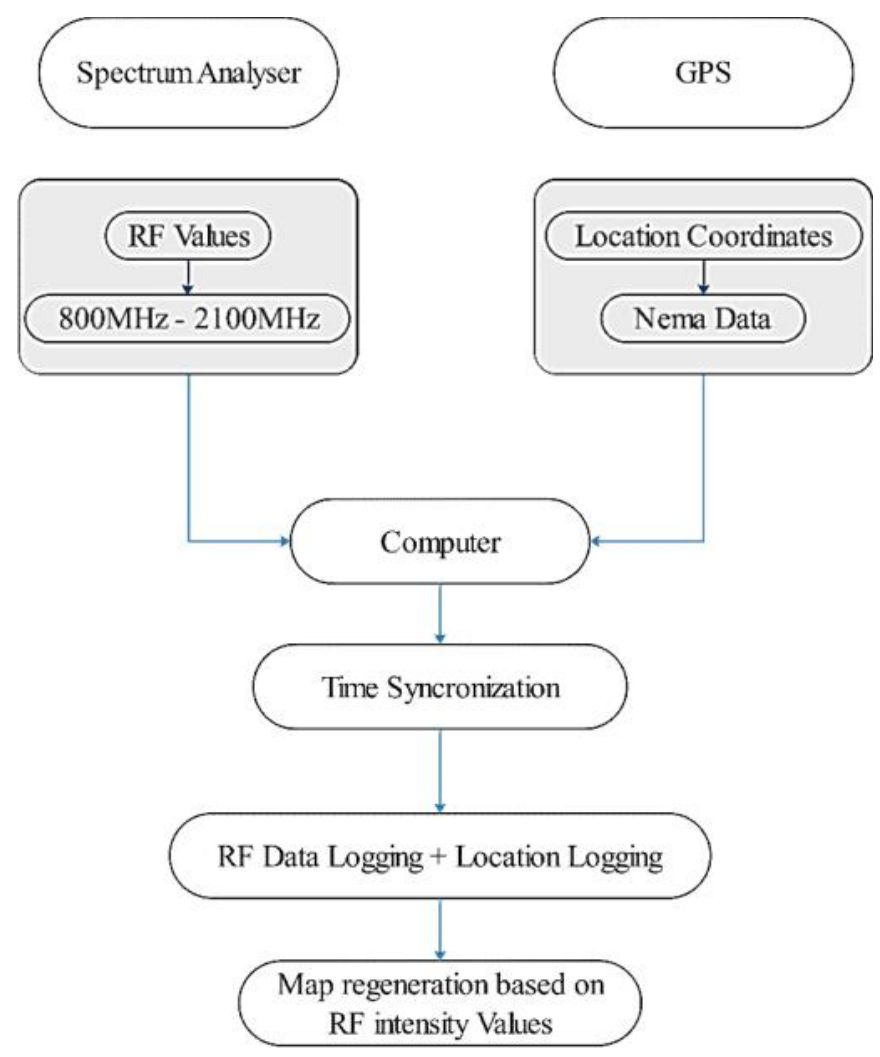

Figure 2: Experimental design setup 


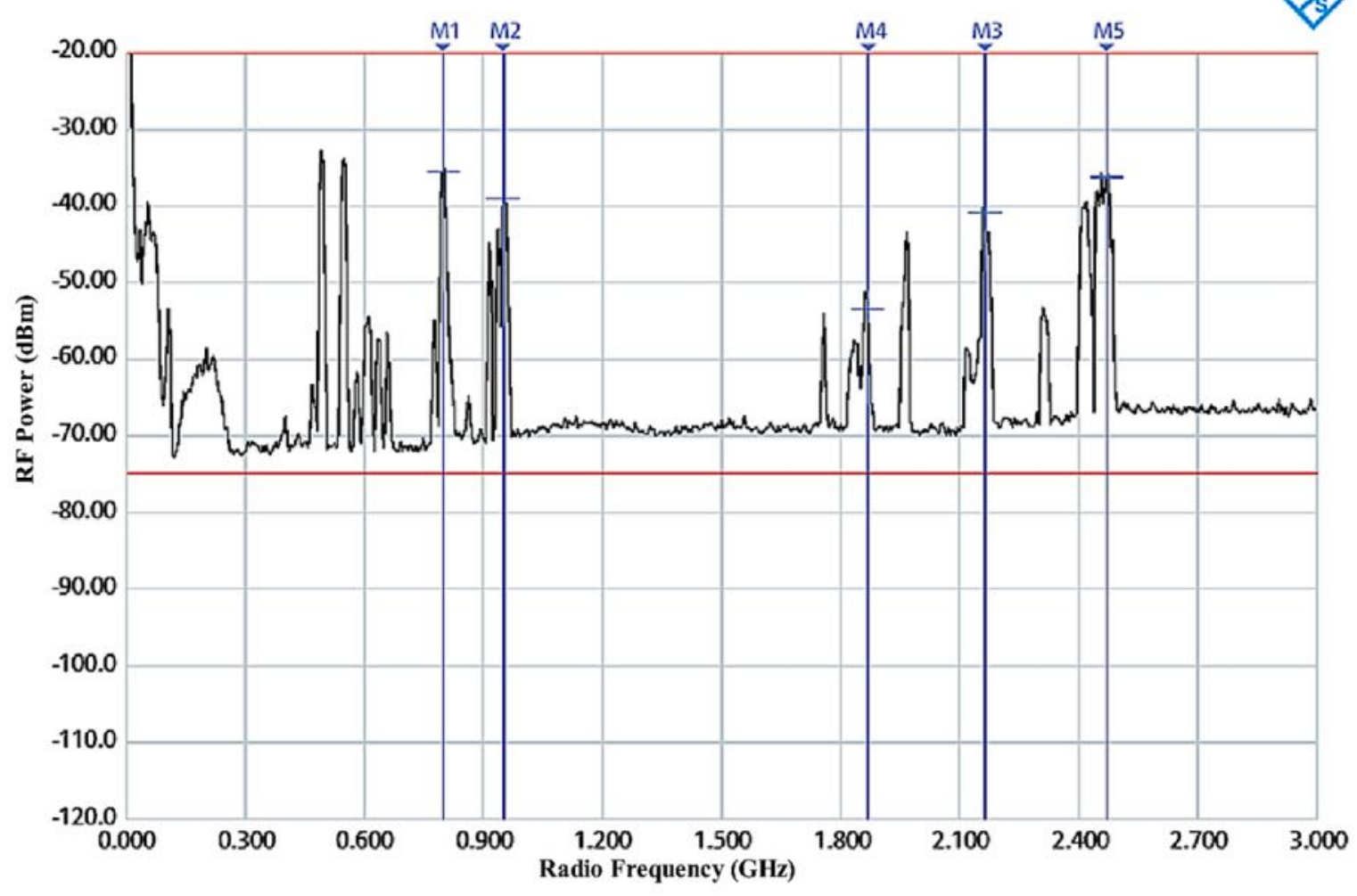

Figure 3: Maximum RF levels at UDSM - CoICT

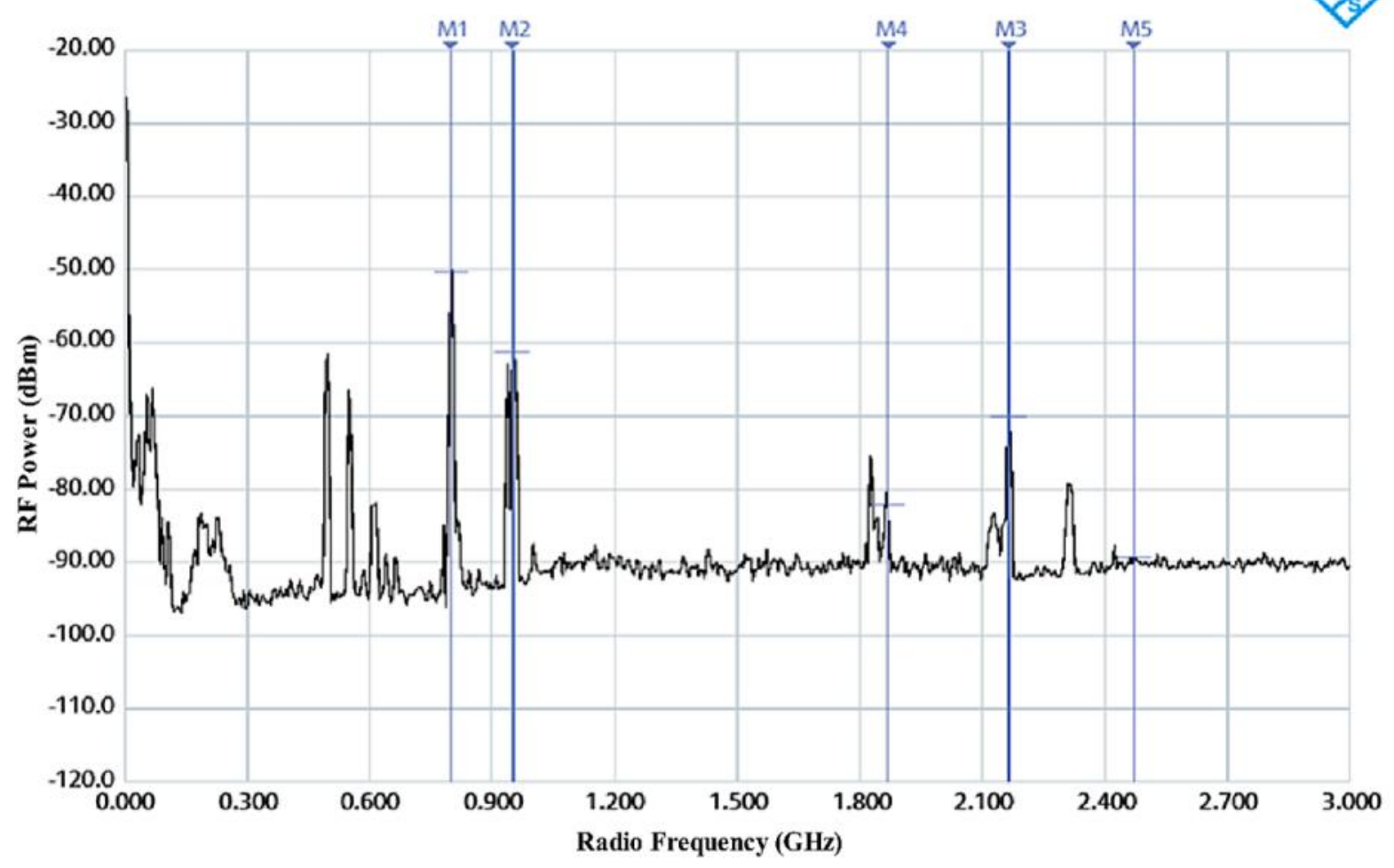

Figure 4: Minimum RF levels at UDSM - CoICT 
Table 1: Average received power

\begin{tabular}{|c|c|c|c|}
\hline Available Frequency (MHz) & $\begin{array}{c}\text { Maximum } \\
\text { Power }(\mathrm{dBm})\end{array}$ & $\begin{array}{c}\text { Minimum } \\
\text { Power }(\mathrm{dBm})\end{array}$ & Average power $(\mathrm{dBm})$ \\
\hline \multirow{4}{*}{800} & -55.46 & -70.97 & \multirow[b]{4}{*}{-63.60} \\
\hline & -53.51 & -94.06 & \\
\hline & -48.25 & -69.91 & \\
\hline & -50.11 & -66.55 & \\
\hline \multirow{4}{*}{950} & -45.91 & -67.31 & \multirow[b]{4}{*}{-61.53} \\
\hline & -41.67 & -70.04 & \\
\hline & -65.37 & -89.88 & \\
\hline & -46.57 & -65.53 & \\
\hline \multirow{4}{*}{2100} & -45.93 & -67.49 & \multirow[b]{4}{*}{-60.82} \\
\hline & -50.62 & -62.80 & \\
\hline & -59.72 & -69.75 & \\
\hline & -59.25 & -71.04 & \\
\hline \multirow{4}{*}{2400} & -24.2 & -65.30 & \multirow[b]{4}{*}{-52.04} \\
\hline & -17.6 & -65.96 & \\
\hline & -41.7 & -70.37 & \\
\hline & -69.68 & -61.57 & \\
\hline
\end{tabular}

Table 2: A 60 second location to RF signal power data

\begin{tabular}{|c|c|c|c|c|c|}
\hline Latitude & Longitude & $\mathbf{8 0 0} \mathbf{~ M H z}$ & $\mathbf{1 8 0 0} \mathbf{~ M H z}$ & $\mathbf{2 1 0 0} \mathbf{~ M H z}$ & $\mathbf{2 4 0 0} \mathbf{~ M H z}$ \\
\hline-6.602997 & 39.123123 & -55 & -75 & -86 & -108 \\
\hline-6.610755 & 39.104663 & -59 & -78 & -71 & -99 \\
\hline-6.619622 & 39.092296 & -48 & -74 & -70 & -42 \\
\hline-6.646222 & 39.086112 & -56 & -81 & -63 & -65 \\
\hline-6.643494 & 39.117718 & -39 & -55 & -60 & -83 \\
\hline-6.619934 & 39.112707 & -40 & -53 & -51 & -56 \\
\hline-6.618911 & 39.130404 & -55 & -60 & -42 & -70 \\
\hline
\end{tabular}

\section{System design and Simulation}

\section{System Design}

The overall system design was based on the Input frequency, Impedance matching topology and Voltage multiplier. Taking into account the factors, frequencies less than $1 \mathrm{GHz}$ are matched using a T-type impedance match topology while frequencies greater than $1 \mathrm{GHz}$ were matched with a Pie type impedance matching, as recommended in the literature (Sun, 1994; Thakuria et al., 2018; Tran et al., 2017). The $\mathrm{T}$ type and Pie type impedance matching topologies were designed based on their input frequencies and input power level, which was a key parameter on circuit optimization on determining component values that will attain maximum power transfer to load. In accordance with Uzun (2019) and Clerckx et al. (2019), it is important to have a simple and yet efficient passive network that accommodates effectively the use of a single frequency.

For matching network designs, values were determined using matching circuit synthesis in ADS 2019. The major advantage of ADS is its unique ability to provide near practical values of any Electromagnetic (EM) design circuitry, which has been widely used in various research activities (Devi et al., 2012; Nimo 
et al., 2015; Ur-Rehman et al., 2017; Uzun, 2019).

Figures 5 and 6 provide ideal design setup for value determination of resistors, capacitors and inductors (RCL) for the appropriate frequency and average power density. Based on the received signal frequency to be matched, impedance measurement values to match the load is crucial, for this case to obtain theoretical values of indictors and capacitors

$$
\begin{aligned}
& Z_{G}=R_{G}+j X_{G} \\
& Z_{L}=R_{L}+j X_{L}
\end{aligned}
$$

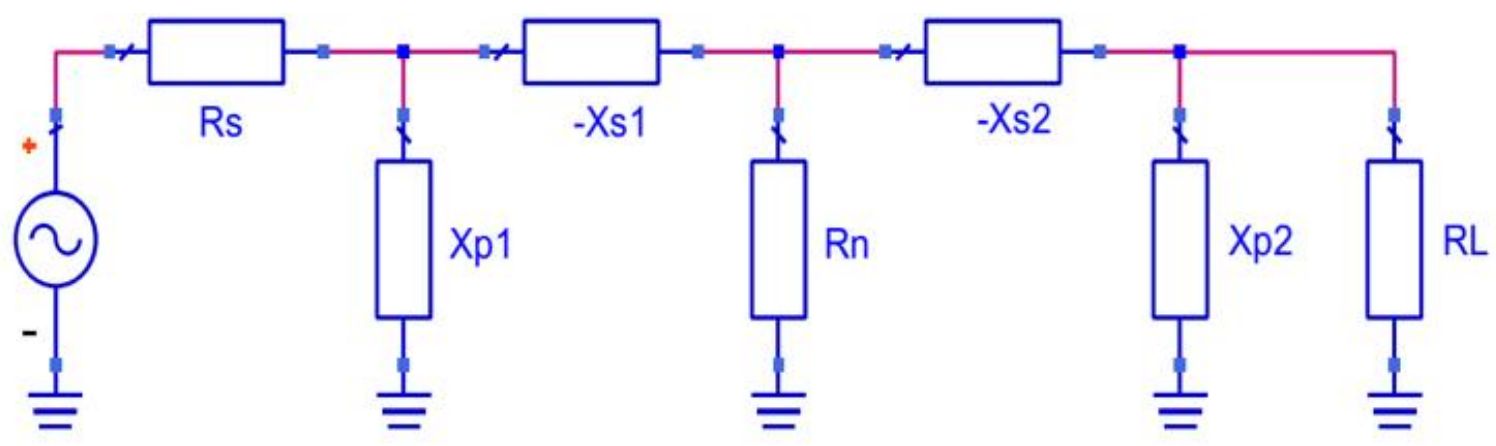

Figure 5: An ideal Pie matching network

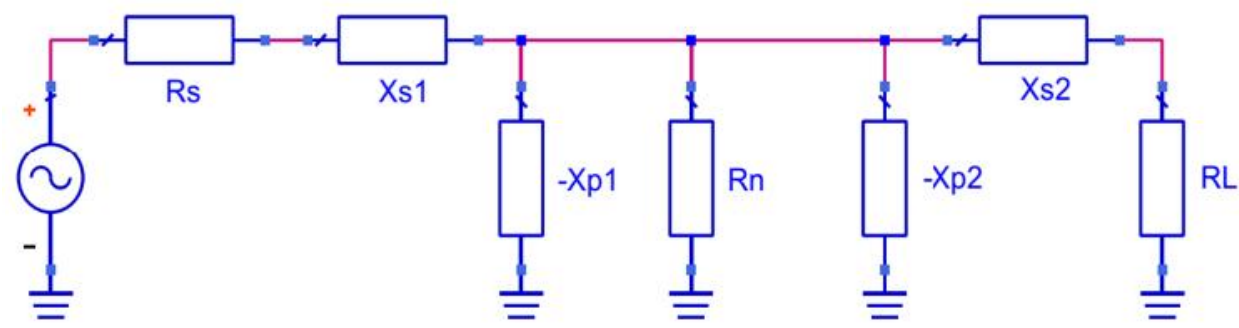

Figure 6: An Ideal T matching network

The impedances described in equations (1) and (2) can be taken as resistors $\mathrm{R}_{\mathrm{S}}$ or $\mathrm{R}_{\mathrm{L}}$ whose reactance is $\mathrm{X}_{\mathrm{G}}$ or $\mathrm{X}_{\mathrm{L}}$ at the desired matching frequency. Assuming an 800 $\mathrm{MHz}$ signal is received, having the antenna and load impedance respectively.

$$
Z_{G}=50+j 6.283 \Omega
$$

$$
Z_{L}=71.701+j 45.216 \Omega
$$

For this case, a Q based algebraic method was preferred as suggested by previous researchers such as, (Nimo et al., 2015; Ur-Rehman et al., 2017). This method can be easily described by the following series to parallel transformation approach. 


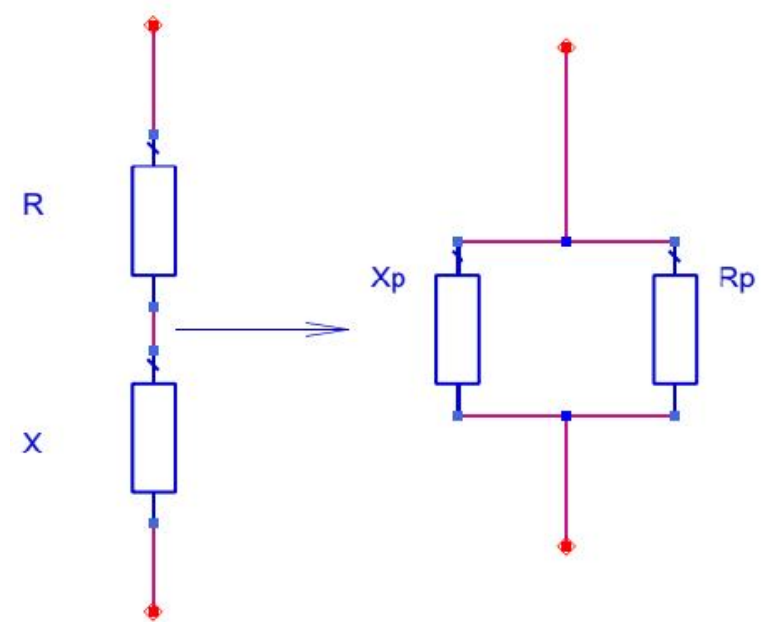

Figure 7: Series to Parallel transformation in passive value determination

The new virtual elements observed in Figure 7 can be determined using equation (5).

$$
\begin{aligned}
& R_{P}=R\left(Q^{2}+1\right) \\
& X_{P}=X\left(\frac{Q^{2}+1}{Q^{2}}\right)
\end{aligned}
$$

Where $Q=\frac{X}{R}$

$Q_{\text {new }}=\sqrt{\frac{R_{G, P}}{R}-1}=\sqrt{\frac{50.789}{3.269}}=3.9416$

$X_{P 1}=\frac{R_{G, P}}{Q_{\text {new }}}=\frac{50.789}{3.9416}=15.199 \Omega$

$X_{S 1}=Q_{\text {new }} x R=3.9416 \times 3.269=12.885 \Omega$

This proposed method has widely been utilized in $\mathrm{Pi}$ and $\mathrm{T}$ matching networks. Assuming that, $\mathrm{Q}=5$, the receivers impedance can be modelled as a resistor having $R_{G_{i} p}=50.789 \Omega$ with an inductor $L_{G, P}=64.326 \mathrm{nH}$ and the load impedance of $R_{L p}=85 \Omega$ with $1 \mathrm{pF}$ capacitor in parallel. The virtual resistor values can be determined, Because $R_{L P}>R_{G, p}$ it is important that $\mathrm{X}_{\mathrm{S} 2}$ and $\mathrm{X}_{\mathrm{P} 2}$ are determined first (Şengül and Yeşilyurt, 2018).

$$
\begin{aligned}
& R=\frac{R_{L, P}}{Q^{2}+1}=\frac{85}{5^{2}+1}=3.269 \ldots \ldots . . . \\
& X_{P 2}=\frac{R_{L, P}}{Q}=\frac{85}{5}=17 \Omega \ldots \ldots \ldots \ldots . . . \\
& X_{S 2}=Q x R=5 \times 3.269=16.345 \Omega
\end{aligned}
$$

Calculating the $\mathrm{Q}$ from the receiver,

Based on the ideal impedance matching networks described in Figures 5 and 6, the approach is theoretically correct on determining lumped components values for pie networks. The same is applied to $\mathrm{T}$ matching networks except that, $R_{G}<R_{L}$ hence $X_{S 1}$ and $X_{P 1}$ are first identified.

After a complete design as described on Figure 8, circuit simulation and optimization were performed on each frequency independently. This utilized two common topologies with different values, the output signals received were integrated to the Villard voltage multiplier, producing an overall output of $149 \mathrm{mV}$. 

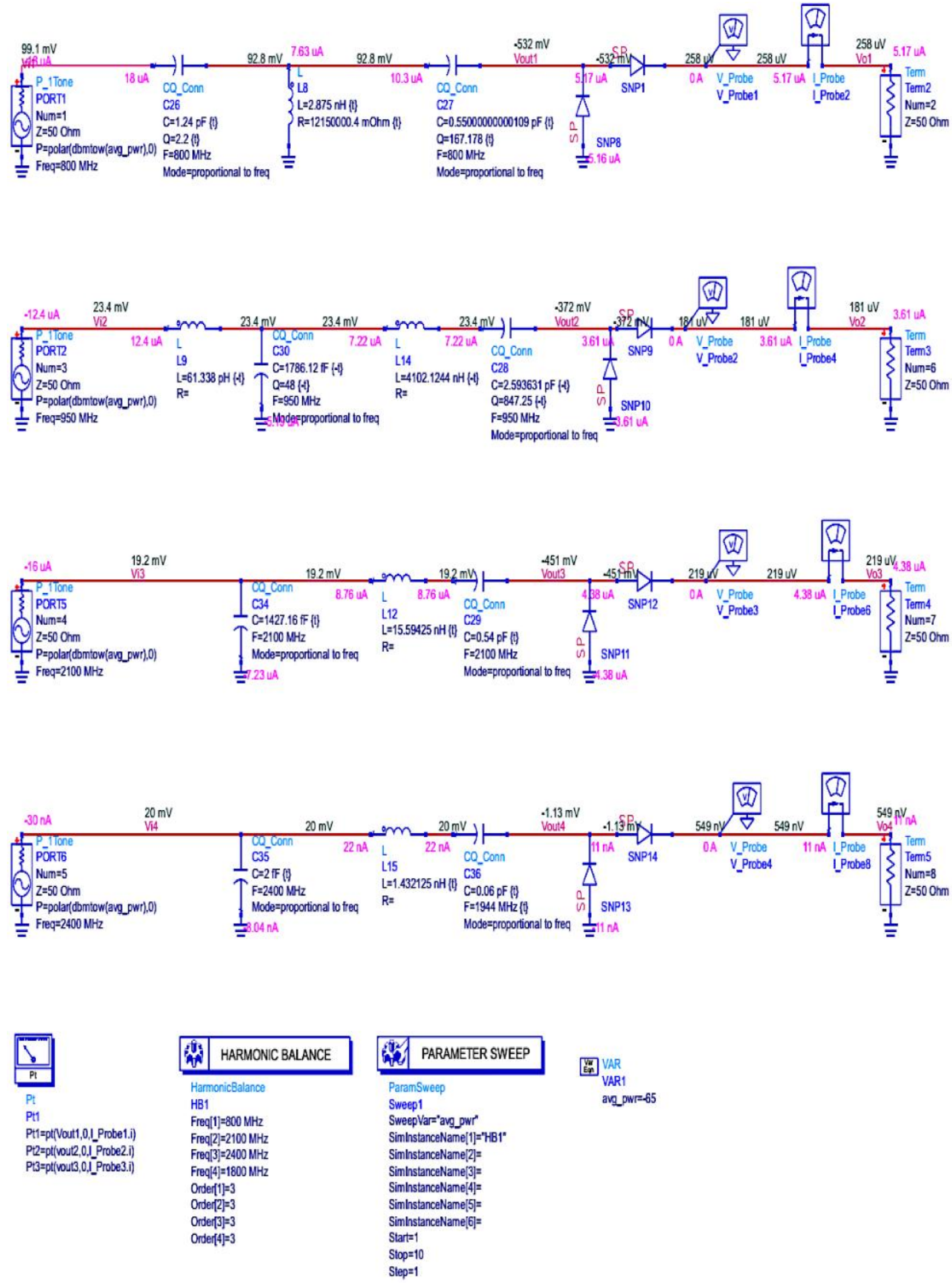

Figure 8: Overall System Design 


\section{RESULTS AND DISCUSSION}

\section{System Design and Simulation}

Figure 8 provides the overall system design showing a one to one, frequency to preferred impedance topology. This investigation provides the maximum achievable power that can be harvested at similar environments using ideal components. Table 3 provides the relationship between the input frequency and output voltages and currents attained from the simulation.

Table 2: Input Frequency with respective output Voltage and Currents

\begin{tabular}{|c|c|c|c|}
\hline Frequency (MHz) & Input Current (uA) & Output Voltage (mV) & Output Current (uA) \\
\hline 800 & 3.251 & 2.417 & 3.415 \\
\hline 1800 & 3.142 & 3.952 & 3.334 \\
\hline 2100 & 2.031 & 5.218 & 2.081 \\
\hline 2400 & 8.405 & 11.325 & 10.208 \\
\hline
\end{tabular}

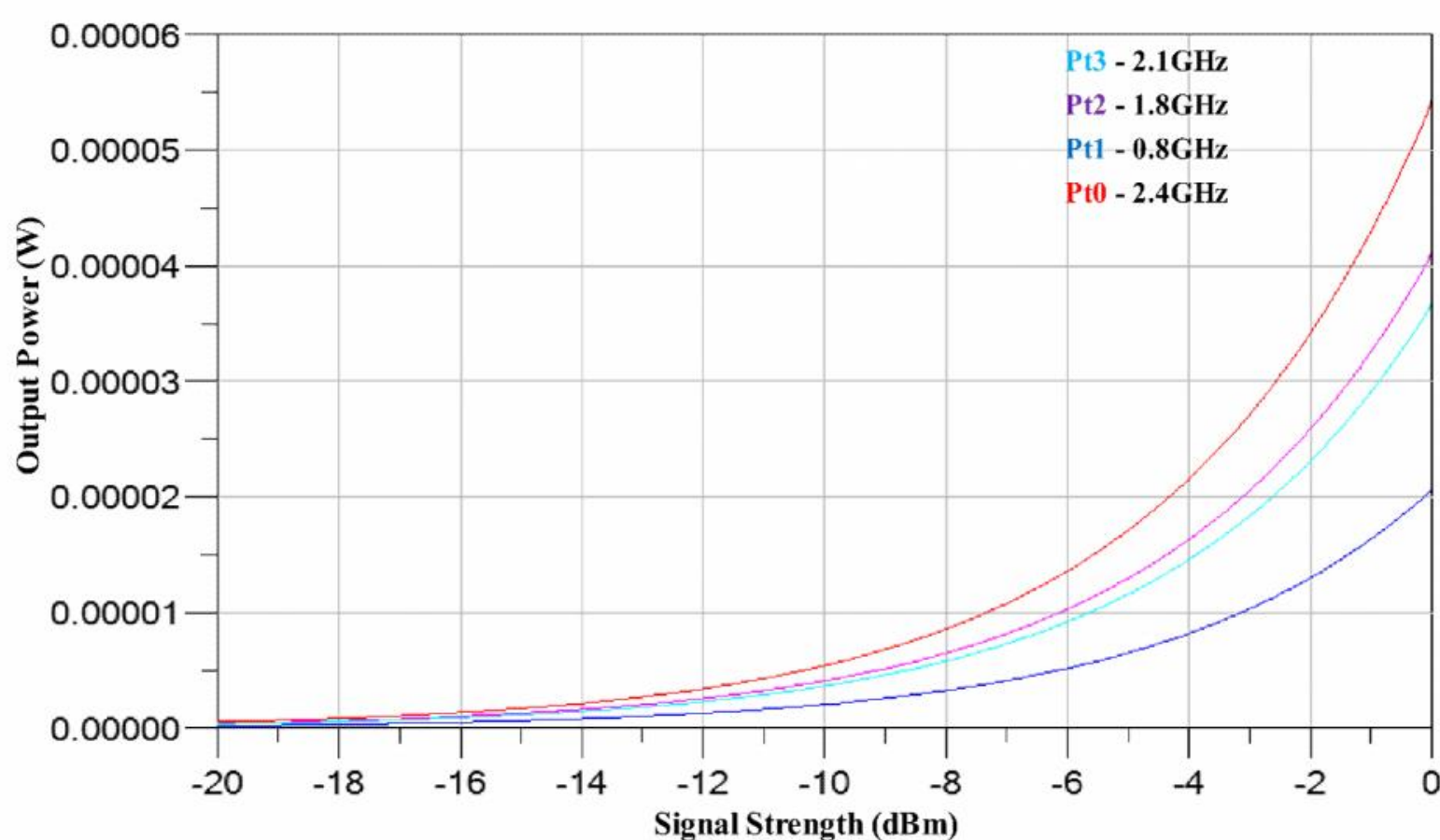

Figure 9: RF Received Signal Strength Vs Output power (W)

Through utilizing narrowband antennas for particular singular frequency reception has shown promising results over time, the capacitor charging time may vary depending on its capacity as larger capacitors will take longer charging time and vice versa. The received RF signals induce an electric and magnetic flux into the system via the designed antenna. It can be observed that there is a small amount of current input due to the nature of RF's being of a low density. For that reason, RF harvesters have limited applicability on devices as they will operate for a short period of time and have longer periods of off-state quietly recharging. As observed, the output current released from the voltage multiplier is slightly greater than the induced current, which is mainly due to the rectification process and the use of BAT 63.03 rectifiers. The output power released by the designed and simulated circuit, can easily be observed that the stronger the signal, the higher is the output 
power. This fading or sometimes termed free space path loss effect is normally observed when moving away from a transmitting source which is expressed by equation (14).

$$
R S S I=10 \log \frac{P_{R X}}{P_{\operatorname{Re} f}}
$$

\section{RF Survey}

From the Dar es Salaam RF survey data in Figure 3 that contained a 60 second location to RF signal power data, a heat map was generated using QGIS to show the RF intensities between $800 \mathrm{MHz}$ to $2400 \mathrm{MHz}$ in Figure 10. The results expressed included 181,000 points with location coordinates (Latitude, Longitude) and $\mathrm{RF}$ received signal strength intensity (RSSI) in $\mathrm{dBm}$.

\section{Challenges}

The main challenges facing RF energy harvesters are low output voltages and currents. To improve such shortcomings there is a huge necessity on improving antennas to meet specific impedance matching topologies so as to assure maximum power transfer. In addition to that, further improvements of voltage multipliers are also inevitable so as to accommodate a wider range of appliances.

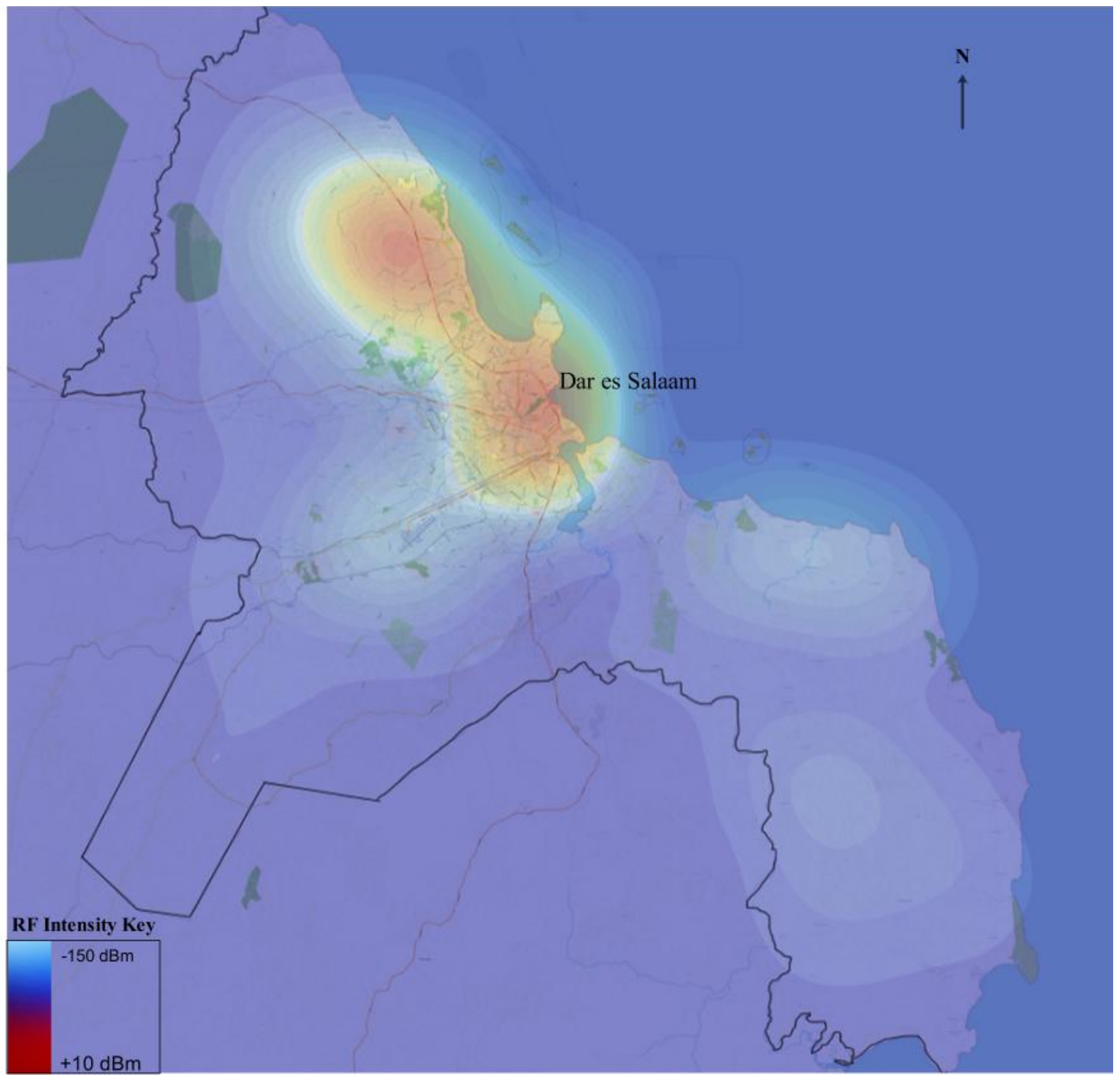

Figure 10: Dar es Salaam City RF Heatmap 


\section{CONCLUSIONS}

This paper discusses the possibilities and challenges of RF energy harvesting in Dar es Salaam City, based upon the RF survey, tools used and their efficiencies. The results obtained revealed a promising future on harvesting ambient RF energy to endlessly power up low power and ultralow power devices throughout its lifetime. To achieve better results, there is a need for more sensitive antennas need to be designed within the narrowband frequency limits. There is also a need for pursuing intensive research on extremely low power (ELP) active and passive devices such as rectifiers and inductors to operate on Pico to Nano voltage scales. The achievements on this will lead to smaller and more efficient electronic components size that will utilize less power compared to the existing ones.

\section{REFERENCES}

Cansiz M., Altinel D. and Kurt G.K. (2019). Efficiency in RF energy harvesting systems: A comprehensive review. Energy, 174: 292-309. https://doi.org/10.1016/j.energy.2019.0 2.100

Clerckx B., Zhang R., Schober R., Ng D.W.K., Kim D.I. and Poor H.V. (2019). Fundamentals of wireless information and power transfer: From RF energy harvester models to signal and system designs. IEEE Journal on Selected Areas in Communications, 37(1): 4-33. https://doi.org/10.1109/JSAC.2018.287 2615

Devi K.K.A., Din N., Chakrabarty C.K. and Sadasivam S. (2012). Design of an RF - DC Conversion Circuit for Energy Harvesting. IEEE International Conference on Electronics Design, Systems and Applications (ICEDSA), 156-161.
10.1109/ICEDSA.2012.6507787

Ejaz W., Naeem M., Shahid A., Anpalagan

A. and Jo M. (2017). Efficient Energy Management for Internet of Things in Smart Cities. IEEE Communications Magazine.

https://doi.org/10.1109/MCOM.2017.16 00218CM

Jose J., George S., Bosco L., Bhandari J., Fernandes F. and Kotrashetti A. (2015). A review of RF energy harvesting systems in India. Proceedings of the International Conference on Technologies for Sustainable Development, ICTSD 2015. https://doi.org/10.1109/ICTSD.2015.70 95838

Khemar A., Kacha A., Takhedmit H. and Abib G. (2018). Design and experiments of a dual-band rectenna for ambient RF energy harvesting in urban environments. IET Microwaves, Antennas \& Propagation, 12(1). https://doi.org/10.1049/ietmap.2016.1040

Marincic A.S. (1982). Nikola Tesla and the Wireless Transmission of Energy. IEEE Transactions on Power Apparatus and Systems. https://doi.org/10.1109/TPAS.1982.317 084

Nimo A., Beckedahl T., Ostertag T. and Reindl L. (2015). Analysis of Passive RF-DC Power Rectification and Harvesting Wireless RF Energy for Micro-watt Sensors. AIMS Energy, 3(2): $\quad$ 184-200. https://doi.org/10.3934/energy.2015.2.1 84

Olgun U., Chen C.C. and Volakis J.L. (2010). Low-profile planar rectenna for batteryless RFID sensors. 2010 IEEE International Symposium on Antennas and Propagation and CNC-USNC/URSI Radio Science Meeting - Leading the Wave, AP-S/URSI 2010. https://doi.org/10.1109/APS.2010.5562 220

QGIS Development Team (2020). QGIS 
Geographic Information System. Open Source Geospatial Foundation Project. http://qgis.osgeo.org

Saini G. and Baghini M.S. (2019). A generic power management circuit for energy harvesters with shared components between the MPPT and regulator. IEEE Transactions on Very Large Scale Integration (VLSI) Systems, 27(3): 535-548. https://doi.org/10.1109/TVLSI.2018.28 85928

Şengül M. and Yeşilyurt G. (2018). Real frequency design of $\mathrm{Pi}$ and $\mathrm{T}$ matching networks with complex terminations. 2017 10th International Conference on Electrical and Electronics Engineering, ELECO 2017, 2018-Janua, 1328-1331.

Shariati N., Rowe W.S.T., Scott J.R. and Ghorbani K. (2015). Multi-service highly sensitive rectifier for enhanced RF energy scavenging. Scientific Reports, 5: 1-9. https://doi.org/10.1038/srep09655

Sun Y. (1994). Practical considerations of impedance matching network design. Sixth International Conference on 'HF Radio Systems and Techniques', 392: 229-233. https://doi.org/10.1049/cp:19940498

Thakuria T., Bezboruah T. and Singh H.K. (2018). Design of an Efficient RF
Energy Harvesting System at $900 \mathrm{MHz}$. 2018 5th International Conference on Signal Processing and Integrated Networks, SPIN 2018, 84-87. https://doi.org/10.1109/SPIN.2018.8474 258

Tran L.-G., Cha H.-K. and Park W.-T. (2017). RF power harvesting: a review on designing methodologies and applications. Micro and Nano Systems Letters, $\quad 5(1)$ : 14. https://doi.org/10.1186/s40486-0170051-0

Ur-Rehman M., Ahmad W., Qureshi M.I. and Khan W.T. (2017). A highly efficient tri band (GSM1800, WiFi2400 and WiFi5000) rectifier for various radio frequency harvesting applications. Progress in Electromagnetics Research Symposium, 2039-2044. https://doi.org/10.1109/PIERS-

FALL.2017.8293473

Uzun Y. (2019). A Comparative Study: Voltage Multipliers for Energy, 61 (January), 12-23. https://doi.org/10.33769/aupse.469183

Valenta C.R. and Durgin G.D. (2014). Harvesting Wireless Power. IEEE Microwave Magazine, 15(4): 108-120. https://doi.org/10.1109/MMM.2014.230 9499 